\title{
On a Reconstruction Algorithm for the Trajectory and Control in a Delay System
}

\author{
M. S. Blizorukova ${ }^{1}$ and V. I. Maksimov ${ }^{1}$ \\ Received June 1, 2011
}

\begin{abstract}
We discuss a problem of the dynamic reconstruction of unmeasured coordinates of the phase vector and unknown controls in nonlinear vector equations with delay. A regularizing algorithm is proposed for the reconstruction of both controls and unmeasured coordinates simultaneously with the processes. The algorithm is stable with respect to information noises and computational errors.
\end{abstract}

Keywords: dynamic reconstruction, method of auxiliary models.

DOI: $10.1134 /$ S0081543813020065

\section{INTRODUCTION AND PROBLEM STATEMENT}

We consider a control system of the form

$$
\begin{gathered}
\dot{x}(t)=f_{1}\left(t, x_{t}(s), y_{t}(s)\right)+f_{2}\left(t, x_{t}(s), y_{t}(s)\right) u(t), \\
\dot{y}(t)=\psi_{1}\left(t, x_{t}(s), y_{t}(s)\right)+\psi_{2}\left(t, y_{t}(s)\right) x(t)
\end{gathered}
$$

with initial conditions

$$
x_{t_{0}}(s)=x_{0}(s) \in C\left(\left[-\tau_{m}^{x}, 0\right] ; \mathbb{R}^{n_{1}}\right), \quad y_{t_{0}}(s)=y_{0}(s) \in C\left(\left[-\tau_{n}^{y}, 0\right] ; \mathbb{R}^{n_{2}}\right) .
$$

Here, $t$ is time from a given interval $T=\left[t_{0}, \vartheta\right]\left(t_{0}<\vartheta<+\infty\right) ; x$ and $y$ are $n_{1}$ - and $n_{2}$-dimensional vectors (which we assume to be columns), respectively, that characterize the state of the system; $u(t)$ is an $r$-dimensional vector of control; and $x_{t}(s)$ and $y_{t}(s)$ are the functions $x_{t}(s)=x(t+s)$ for $s \in\left[-\tau_{m}^{x}, 0\right]$ and $y_{t}(s)=y(t+s)$ for $s \in\left[-\tau_{n}^{y}, 0\right]$. The structure of the vector functions $f_{1}$ and $\psi_{1}$ and of the matrix functions $f_{2}$ and $\psi_{2}$ is clarified below.

Initial state (1.3) is assumed to be a Lipschitz function. In what follows, the symbol $P$ denotes a fixed compact set in $\mathbb{R}^{r}$ (the control resources); $x_{0}(s)$ and $y_{0}(s)$ are known fixed functions. Any (Lebesgue) measurable function $u(\cdot)$ from the set $P(\cdot)=\left\{u(\cdot) \in L_{2}\left(T ; \mathbb{R}^{r}\right): u(t) \in P\right.$ for a.a. $\left.t \in T\right\}$ is called a control, and the solution $z(\cdot)=\{x(\cdot), y(\cdot)\}$ (in the sense of Carathéodory) of system of equations (1.1), (1.2) with initial condition (1.3) is called a motion of the system generated by the control $u(\cdot)$ (and starting from the initial state $\left.\left\{x_{0}(s), y_{0}(s)\right\}\right)$.

\footnotetext{
${ }^{1}$ Institute of Mathematics and Mechanics, Ural Branch of the Russian Academy of Sciences, ul. S. Kovalevskoi 16, Yekaterinburg, 620990 Russia; Graduate School of Economics and Management, Ural Federal University, ul. Mira 19, Yekaterinburg, 620002 Russia email: msb@imm.uran.ru, maksimov@imm.uran.ru
} 
Let $u(\cdot) \in P(\cdot)$ be a control realized on the time interval $T$, and let $z(\cdot)=\{x(\cdot), y(\cdot)\}$ be the motion generated by it. Assume that a part of the current state vector $\left\{x\left(\tau_{i}\right), y\left(\tau_{i}\right)\right\}$, namely, the vector $y\left(\tau_{i}\right)$, is measured during the process at sufficiently frequent times $\tau_{i}$ from $T$. The measurement results $\xi^{h}\left(\tau_{i}\right)$ are inaccurate; they satisfy the inequality

$$
\left|\xi^{h}\left(\tau_{i}\right)-y\left(\tau_{i}\right)\right| \leq h,
$$

where $h$ is small. Here, the symbol $|\cdot|$ denotes the Euclidean norm. The problem consists in constructing an algorithm for the reconstruction of the unmeasured component $x(\cdot)$ of the state vector and the control $u(\cdot)$ in real time from the current measurements $\xi^{h}\left(\tau_{i}\right)$. Since it is impossible to reconstruct their exact values (because $y(\cdot)$ is measured with error), we actually require that the algorithm form (in real time) some approximations $v^{h}(\cdot)$ and $u^{h}(\cdot)$ that are close to $x(\cdot)$ and $u(\cdot)$. More exactly, the standard deviation of $v^{h}(\cdot)$ from $x(\cdot)$

$$
\left|v^{h}(\cdot)-x(\cdot)\right|_{L_{2}(T)}^{2}=\int_{t_{0}}^{\vartheta}\left|v^{h}(t)-x(t)\right|^{2} d t
$$

and the deviation of $v^{h}(\cdot)$ from $u(\cdot)$

$$
\left|u(\cdot)-u^{h}(\cdot)\right|_{L_{2}(T)}^{2}=\int_{t_{0}}^{\vartheta}\left|u(t)-u^{h}(t)\right|^{2} d t
$$

must be arbitrarily small for sufficiently small measurement error $h$.

This problem belongs to the class of inverse problems of control system dynamics (the input is reconstructed from measurements of the output). A posteriori formulations of inverse problems were studied by many authors [1-5]. In [6], a method of dynamic (positional) reconstruction of the input in a finite-dimensional control-affine dynamic system was proposed. The method is based on the ideas from the theory of positional control $[7,8]$ and on the smoothing functional method and the residual method known in the theory of ill-posed problems [1]. For systems described by ordinary differential equations, this method was developed in $[6,15,16]$. The case of measuring all the coordinates of the state vector was considered in [6], and the case of measurements of type (1.4) was studied in $[15,16]$ under some special constraints on the dynamics of the system. In [9-14], the method was developed for various classes of delay systems.

Let elements of the vector function $f_{1}(\cdot)$ and of the matrix function $f_{2}(\cdot)$ have the form

$$
\begin{gathered}
g\left(t, x_{t}(s), y_{t}(s)\right)=g\left(t, x(t), x\left(t-\tau_{1}^{x}\right), \ldots, x\left(t-\tau_{m}^{x}\right), y(t), y\left(t-\tau_{1}^{y}\right), \ldots, y\left(t-\tau_{n}^{y}\right)\right), \\
0<\tau_{1}^{x}<\tau_{2}^{x}<\ldots<\tau_{m}^{x}<+\infty, \quad 0<\tau_{1}^{y}<\tau_{2}^{y}<\ldots<\tau_{n}^{y}<+\infty, \\
g(\cdot)=f_{1 i}(\cdot) \text { for } i \in\left[1: n_{1}\right], \quad g(\cdot)=f_{2 i j}(\cdot) \text { for } i \in\left[1: n_{1}\right], j \in[1: r],
\end{gathered}
$$

and satisfy the Lipschitz condition

$$
\begin{gathered}
\left|g\left(t_{1}, x_{0}^{(1)}, x_{1}^{(1)}, \ldots, x_{m}^{(1)}, y_{0}^{(1)}, y_{1}^{(1)}, \ldots, y_{n}^{(1)}\right)-g\left(t_{2}, x_{0}^{(2)}, x_{1}^{(2)}, \ldots, x_{m}^{(2)}, y_{0}^{(2)}, y_{1}^{(2)}, \ldots, y_{n}^{(2)}\right)\right| \\
\leq c_{1}\left(\left|t_{2}-t_{1}\right|+\sum_{i=0}^{m}\left|x_{i}^{(1)}-x_{i}^{(2)}\right|+\sum_{j=0}^{n}\left|y_{j}^{(1)}-y_{j}^{(2)}\right|\right)
\end{gathered}
$$


Let elements of the matrix function $\psi_{2}(\cdot)$ and of the vector-function $\psi_{1}(\cdot)$ have similar properties:

$$
\begin{gathered}
g_{2}(\cdot)=\psi_{2 i j}(\cdot) \text { for } i \in\left[1: n_{2}\right], j \in\left[1: n_{1}\right] ; \quad g_{2}\left(t, y_{t}(s)\right)=g_{2}\left(t, y(t), y\left(t-\tau_{1}^{y}\right), \ldots, y\left(t-\tau_{n}^{y}\right)\right) ; \\
g_{1}(\cdot)=\psi_{1 i}(\cdot) \text { for } i \in\left[1: n_{2}\right], \\
g_{1}\left(t, x_{t}(s), y_{t}(s)\right)=g_{1}\left(t, x\left(t-\tau_{1}^{x}\right), \ldots, x\left(t-\tau_{m}^{x}\right), y(t), y\left(t-\tau_{1}^{y}\right), \ldots, y\left(t-\tau_{n}^{y}\right)\right) .
\end{gathered}
$$

Here, the elements $g_{1}(\cdot)$ and $g_{2}(\cdot)$ satisfy the conditions

$$
\begin{gathered}
\left|g_{2}\left(t_{1}, y_{0}^{(1)}, y_{1}^{(1)}, \ldots, y_{n}^{(1)}\right)-g_{2}\left(t_{2}, y_{0}^{(1)}, y_{1}^{(2)}, \ldots, y_{n}^{(2)}\right)\right| \leq C_{1}\left(\left|t_{2}-t_{1}\right|+\sum_{j=0}^{n}\left|y_{j}^{(1)}-y_{j}^{(2)}\right|\right), \\
\left|g_{1}\left(t_{1}, x_{1}^{(1)}, \ldots, x_{m}^{(1)}, y_{0}^{(1)}, y_{1}^{(1)}, \ldots, y_{n}^{(1)}\right)-g_{1}\left(t_{2}, x_{1}^{(2)}, \ldots, x_{m}^{(2)}, y_{0}^{(2)}, y_{1}^{(2)}, \ldots, y_{n}^{(2)}\right)\right| \\
\leq d_{1}\left(\left|t_{2}-t_{1}\right|+\sum_{i=1}^{m}\left|x_{i}^{(1)}-x_{i}^{(2)}\right|+\sum_{j=0}^{n}\left|y_{j}^{(1)}-y_{j}^{(2)}\right|\right) .
\end{gathered}
$$

In (1.7)-(1.9) and below, the symbol $|\cdot|$ denotes the Euclidean norm, the corresponding matrix norm, and the absolute value of a number. Under the above conditions, for every initial state (1.3) and control $u(\cdot) \in P(\cdot)$, there exists a unique solution of system (1.1), (1.2). In what follows, we assume $\tau_{m}^{x}=\tau_{n}^{y}=\tau$ for simplicity.

Let us describe the scheme of the algorithm that solves the problem under consideration. Denote by $\xi^{h}(\cdot)$ a function $\xi^{h}(t), t \in\left[t_{0}-\tau, \vartheta\right]$, such that $\xi^{h}(t)=y_{0}\left(t-t_{0}\right)$ for $t \in\left[t_{0}-\tau, t_{0}\right)$ and $\xi^{h}(t)=\xi^{h}\left(\tau_{i}\right)$ for $t \in\left[\tau_{i}, \tau_{i+1}\right), i \in[0: q-1]$, where $\tau_{i}=\tau_{h, i}, q=q_{h}$, and $\xi^{h}\left(\tau_{i}\right)$ satisfies (1.4). For given $h \in(0,1)$, fix the points

$$
\tau_{i}=\tau_{h, i}, \quad i=0,1, \ldots, \quad q=q_{h}, \quad t_{0}=\tau_{0}<\tau_{1}<\ldots<\tau_{q}=\vartheta,
$$

of the time interval $T$. For simplicity, assume that $\tau_{i}-\tau_{i-1}=\delta=\delta(h)$. Thus, for every $h$, the uniform grid $\Delta_{h}=\left\{\tau_{h, i}\right\}_{i=0}^{q_{h}}$ with step $\delta=\delta(h)$ is chosen on $T$ (see (1.10)). Next, we introduce a control system of the form

$$
\begin{gathered}
\dot{w}^{h}(t)=\rho\left(\tau_{i}, v_{\tau_{i}}^{h}(s), \xi_{\tau_{i}}^{h}(s), u_{i}^{h}, w_{\tau_{i}}^{h}(s)\right), \\
\tau_{i}<t \leq \tau_{i+1}, \quad i=0,1, \ldots, m-1, \quad w^{h}\left(t_{0}+s\right)=w_{0}^{h}(s) ;
\end{gathered}
$$

this system is called a model. Here, $w^{h}(t)$ is the finite-dimensional state vector of the model at time $t ; w_{0}^{h}(s)=\left\{w_{0 x}^{h}(s), w_{0 y}^{h}(s)\right\} \in L_{\infty}\left([-\tau, 0] ; \mathbb{R}^{n_{1}+n_{2}}\right)$ is the initial state of the model; $w_{\tau_{i}}^{h}(s)=$ $w^{h}\left(\tau_{i}+s\right) \in \mathbb{R}^{n_{1}+n_{2}}$ for $s \in[-\tau, 0]$; and $v_{i}^{h} \in \mathbb{R}^{n_{1}}$ and $u_{i}^{h} \in \mathbb{R}^{r}$ are the finite-dimensional feedback controls $[7,8]$ in the model generated at time $\tau_{i}$ :

$$
\begin{gathered}
v_{i}^{h}=V\left(\tau_{i}, w_{\tau_{i}}^{h}(s), \xi_{\tau_{i}}^{h}(s)\right), \\
u_{i}^{h}=U\left(\tau_{i}, w_{\tau_{i}}^{h}(s), \xi_{\tau_{i}}^{h}(s), v_{\tau_{i}}^{h}(s)\right),
\end{gathered}
$$

where $\tau_{i}=\tau_{h, i} \in \Delta_{h}, v_{t}^{h}\left(t_{0}+s\right)=x\left(t_{0}+s\right)$ for $s \in[-\tau, 0]$, and $v_{t}^{h}(s)=v^{h}(t+s)$ for $s \in[-\tau, 0]$, $t \geq t_{0}$. The functions $V(\cdot)$ and $U(\cdot)$ are called strategies. The model operates in "real time," i.e., synchronously with system (1.1), (1.2). In the process of its operation, piecewise constant controls $v^{h}(\cdot)$ and $u^{h}(\cdot)$ are formed:

$$
v^{h}(t)=v_{i}^{h}, \quad u^{h}(t)=u_{i}^{h}, \quad \tau_{i}<t \leq \tau_{i+1}, \quad i=0,1, \ldots, q-1 .
$$

They are the required approximations of the functions $x(\cdot)$ and $u(\cdot)$. 


\section{RECONSTRUCTION OF UNKNOWN COORDINATES}

Let us describe the algorithm for the dynamic reconstruction of the unknown coordinate $x(\cdot)$. We will specify the rules for choosing the strategy $V(1.12)$ and model (1.11). This will allow us to construct the function $v^{h}(\cdot)$, which approximates $x(\cdot)$ (see (1.5)).

Fix the value of measurement error $h \in(0,1)$ and the family of partitions $\Delta_{h}$ (1.10) of the interval $T$. Denote by $Z(T)$ the bundle of solutions of system (1.1), (1.2) with initial condition (1.3); i.e., $Z(T)=\left\{z(\cdot)=z\left(\cdot ; t_{0}, z_{0}(s), u(\cdot)\right): u(\cdot) \in P(\cdot)\right\}$. Consider a model described by the equation

$$
\begin{gathered}
\dot{w}^{(1)}(t)=F_{1}\left(\tau_{i}, v_{\tau_{i}}^{h}(s), \xi_{\tau_{i}}^{h}(s), w^{(1)}\left(\tau_{i}\right)\right), \\
F_{1}\left(\tau_{i}, v_{\tau_{i}}^{h}(s), \xi_{\tau_{i}}^{h}(s), w^{(1)}\left(\tau_{i}\right)\right)=\psi_{1}\left(\tau_{i}, v_{\tau_{i}}^{h}(s), \xi_{\tau_{i}}^{h}(s)\right) \\
+\psi_{2}\left(\tau_{i}, \xi_{\tau_{i}}^{h}(s)\right) v_{i}^{h}+2\left(\xi^{h}\left(\tau_{i}\right)-w^{(1)}\left(\tau_{i}\right)\right), \quad w^{(1)} \in \mathbb{R}^{n_{2}}, \quad t \in\left[\tau_{i}, \tau_{i+1}\right),
\end{gathered}
$$

with initial condition $w_{t_{0}}^{(1)}(s)=y\left(t_{0}+s\right), s \in[-\tau, 0), w^{(1)}\left(t_{0}\right)=\xi^{h}\left(t_{0}\right)$. The solution of this equation $w^{(1)}(\cdot)=w^{(1)}\left(\cdot ; t_{0}, w_{t_{0}}^{(1)}(s), v^{h}(\cdot)\right)$ is understood in the sense of Carathéodory.

Assume that $\Delta^{(j)}=\left[t_{j}, t_{j+1}\right], t_{j}=t_{0}+\tau_{1}^{x} j, l$ is the integer part of the number $\tau / \tau_{1}^{x}, j_{*}=$ $\max \left\{j: t_{j}<\vartheta\right\}$, and $g_{j}(h)=h^{(1 / 3)^{j}}$ for $j \in\left[1: j_{*}\right]$. Below, we assume for simplicity that the partitions $\Delta_{h}$ are chosen so that $t_{j} \in \Delta_{h}$. The strategy $V(1.12)$ for $\tau_{i} \in\left[t_{j}, t_{j+1}\right) \cap T$ is specified as follows:

$$
\begin{gathered}
V\left(\tau_{i}, w_{\tau_{i}}^{(1)}(s), \xi_{\tau_{i}}^{h}(s)\right)=V_{j}\left(\tau_{i}, w_{\tau_{i}}^{(1)}(s), \xi_{\tau_{i}}^{h}(s)\right) \\
=\arg \min \left\{2\left(l_{i}, \psi_{2}\left(\tau_{i}, \xi_{\tau_{i}}^{h}(s)\right) v\right)+\alpha_{j}|v|^{2}: v \in S(A)\right\} .
\end{gathered}
$$

Here, $\alpha_{j}$ is a parameter, $j \in\left[0: j_{*}\right], S(A) \subset \mathbb{R}^{n_{1}}$ is a ball of radius $A=\sup \left\{|x(\cdot)|_{C\left(T ; \mathbb{R}^{\left.n_{1}\right)}\right.}: z(\cdot)=\right.$ $\{x(\cdot), y(\cdot)\} \in Z(T)\}<+\infty$ centered at zero, and $l_{i}=w^{(1)}\left(\tau_{i}\right)-\xi^{h}\left(\tau_{i}\right)$.

Assume that the following condition is satisfied.

Condition 1. Assume that $n_{1} \leq n_{2}$ and there exists $c_{*}>0$ such that the matrix $\psi_{2}\left(t, y_{t}(s)\right)$ has a minor of $n_{1}$ th order with the following property: the $n_{1} \times n_{1}$-matrix $\bar{\psi}_{2}(t)=\bar{\psi}_{2}\left(t, y_{t}(s)\right)$ corresponding to this minor satisfies the condition $\left|\bar{\psi}_{2}(t) x\right| \geq c_{*}|x|$ for all $t \in T$ and $x \in \mathbb{R}^{n_{1}}$.

Choose the parameter $\alpha_{j}$ as follows:

$$
\alpha_{0}=C h^{2 / 3}, \quad \alpha_{j}=C g_{j}^{2 / 3}(h), \quad j \geq 1, \quad C=\text { const }>0 .
$$

Theorem 1. Let $\delta=\delta(h) \leq h$. Then,

$$
\left|v^{h}(\cdot)-x(\cdot)\right|_{L_{2}\left(\Delta^{(j-1)} ; \mathbb{R}^{\left.n_{1}\right)}\right.}^{2} \leq c_{j} g_{j}(h), \quad j \in\left[1: j_{*}\right] .
$$

Before starting to prove the theorem, we give auxiliary statements. Theorem 1 will follow from Lemma 5. Consider the two systems

$$
\dot{p}(t)=f_{1}(t)+f_{2}(t) u_{1}(t), \quad \dot{q}(t)=F_{1}(t)+F_{2}(t) u_{2}(t), \quad t \in T,
$$

where $p(t), q(t) \in \mathbb{R}^{n}, f_{1}(\cdot), F_{1}(\cdot) \in L_{2}\left(T ; \mathbb{R}^{n}\right), f_{2}(\cdot), F_{2}(\cdot) \in L_{2}\left(T ; \mathbb{R}^{n \times r}\right), u_{1}(\cdot), u_{2}(\cdot) \in L_{2}\left(T ; \mathbb{R}^{r}\right)$, and $\left|u_{p}(\cdot)\right|_{L_{\infty}\left(T ; \mathbb{R}^{r}\right)} \leq K$ for $p=1,2$.

Introduce the notation: $\Delta_{*}^{(j)}=\left[t_{j}^{*}, t_{j+1}^{*}\right] \cap T$ and $t_{j}^{*}=t_{0}+\tau_{*} j$ for $j \in\left[0: j_{0}\right], \Delta^{(-1)}=\left[t_{0}-\tau_{*}, t_{0}\right]$, $\tau_{*}=$ const $\in\left(0, \vartheta-t_{0}\right)$, and $j_{0}=\max \left\{j: t_{j}^{*} \leq \vartheta\right\}$. Assume that $r \leq n$ and there exists $c>0$ such 
that the matrix $f_{2}(t)$ has a minor of $r$ th order with the following property: the $r \times r$-matrix $\bar{f}_{2}(t)$ corresponding to this minor is such that $\left|\bar{f}_{2}(t) u\right| \geq c|u|$ for all $t \in T$ and $u \in \mathbb{R}^{r}$.

Lemma 1. Suppose that $t \rightarrow\left(\bar{f}_{2}(t)\right)^{-1} u_{1}(t)$ is a function of bounded variation on $T$,

$$
\begin{gathered}
\left|f_{1}(\cdot)-F_{1}(\cdot)\right|_{L_{2}\left(\Delta_{*}^{(j)} ; \mathbb{R}^{n}\right)}^{2} \leq a_{1}^{(j)}, \\
\left|f_{2}(\cdot)-F_{2}(\cdot)\right|_{L_{2}\left(\Delta_{*}^{(j)} ; \mathbb{R}^{n \times r}\right)}^{2} \leq a_{2}^{(j)}, \\
\left|p\left(t_{j}^{*}\right)-q\left(t_{j}^{*}\right)\right|^{2} \leq a_{4}^{(j)}, \\
|p(t)-q(t)|^{2}+\tilde{\alpha}_{j} \int_{t_{j}^{*}}^{t}\left\{\left|u_{2}(\nu)\right|^{2}-\left|u_{1}(\nu)\right|^{2}\right\} d \nu \leq a_{3}^{(j)},
\end{gathered}
$$

where $t \in\left[t_{j}^{*}, t_{j+1}^{*}\right]$ and $\tilde{\alpha}_{j}=$ const $\in(0,+\infty)$. Then,

$$
\mu^{(j)} \equiv\left|u_{1}(\cdot)-u_{2}(\cdot)\right|_{L_{2}\left(\Delta_{*}^{(j)} ; \mathbb{R}^{r}\right)}^{2} \leq K_{j}\left\{\sum_{l=1}^{4}\left(a_{l}^{(j)}\right)^{1 / 2}+\tilde{\alpha}_{j}^{1 / 2}\right\}+a_{3}^{(j)} / \tilde{\alpha}_{j}
$$

Proof. Let $t \in \Delta_{*}^{(j)}$. Then, by (2.4)-(2.7), we have the estimate

$$
\begin{gathered}
\left|\int_{t_{j}^{*}}^{t} \bar{f}_{2}(\nu)\left\{u_{1}(\nu)-u_{2}(\nu)\right\} d \nu\right| \leq\left|\int_{t_{j}^{*}}^{t} f_{2}(\nu)\left\{u_{1}(\nu)-u_{2}(\nu)\right\} d \nu\right| \\
=\left|\int_{t_{j}^{*}}^{t}\left\{\dot{p}(\nu)-f_{1}(\nu)-f_{2}(\nu) u_{2}(\nu)\right\} d \nu\right|=\left|\int_{t_{j}^{*}}^{t}\left\{\dot{p}(\nu)-\dot{q}(\nu)+F_{1}(\nu)-f_{1}(\nu)+\left(F_{2}(\nu)-f_{2}(\nu)\right) u_{2}(\nu)\right\} d \nu\right| \\
\leq\left(a_{3}^{(j)}+2 \tau_{*} K^{2} \tilde{\alpha}_{j}\right)^{1 / 2}+\left(a_{4}^{(j)}\right)^{1 / 2}+\tau_{*}^{1 / 2}\left\{\left(a_{1}^{(j)}\right)^{1 / 2}+K\left(a_{2}^{(j)}\right)^{1 / 2}\right\} .
\end{gathered}
$$

Using relation (2.7), we derive the inequality

$$
\begin{gathered}
\mu^{(j)}=\left|u_{1}(\cdot)\right|_{L_{2}\left(\Delta_{*}^{(j)} ; \mathbb{R}^{r}\right)}^{2}-2\left(u_{1}(\cdot), u_{2}(\cdot)\right)_{L_{2}\left(\Delta_{*}^{(j)} ; \mathbb{R}^{r}\right)} \\
+\left|u_{2}(\cdot)\right|_{L_{2}\left(\Delta_{*}^{(j)} ; \mathbb{R}^{r}\right)}^{2} \leq 2\left|u_{1}(\cdot)\right|_{L_{2}\left(\Delta_{*}^{(j)} ; \mathbb{R}^{r}\right)}^{2}-2\left(u_{1}(\cdot), u_{2}(\cdot)\right)_{L_{2}\left(\Delta_{*}^{(j)} ; \mathbb{R}^{r}\right)}+a_{3}^{(j)} / \tilde{\alpha}_{j} .
\end{gathered}
$$

Hence,

$$
\begin{gathered}
\mu^{(j)} \leq 2 \int_{t_{j}^{*}}^{t_{j+1}^{*}}\left(u_{1}(\nu)-u_{2}(\nu), u_{1}(\nu)\right)_{\mathbb{R}^{r}} d \nu+a_{3}^{(j)} / \tilde{\alpha}_{j} \\
=2 \int_{t_{j}^{*}}^{t_{j+1}^{*}}\left(\bar{f}_{2}(\nu)\left(u_{1}(\nu)-u_{2}(\nu)\right), \bar{f}_{2}^{-1}(\nu) u_{1}(\nu)\right)_{\mathbb{R}^{r}} d \nu+a_{3}^{(j)} / \tilde{\alpha}_{j} .
\end{gathered}
$$


Therefore, in view of (2.8), (2.9), and the results of [17], we obtain

$$
\begin{gathered}
\mu^{(j)} \leq\left\{\left(a_{3}^{(j)}+2 \tau_{*} K^{2} \tilde{\alpha}_{j}\right)^{1 / 2}+\left(a_{4}^{(j)}\right)^{1 / 2}+\tau_{*}^{1 / 2}\left(\left(a_{1}^{(j)}\right)^{1 / 2}+\left(a_{2}^{(j)}\right)^{1 / 2}\right)\right\} \\
\times\left(\sup _{t \in \Delta_{*}^{(j)}}\left|\bar{f}_{2}^{-1}(t) u_{1}(t)\right|+\operatorname{var}_{\Delta_{*}^{(j)}}\left(\bar{f}_{2}^{-1}(\cdot) u_{1}(\cdot)\right)\right)+a_{3}^{(j)} / \tilde{\alpha}_{j} \leq K_{j}\left\{\sum_{l=1}^{4}\left(a_{l}^{(j)}\right)^{1 / 2}+\tilde{\alpha}_{j}^{1 / 2}\right\}+a_{3}^{(j)} / \tilde{\alpha}_{j} .
\end{gathered}
$$

The lemma is proved.

Lemma 2. The bundle of solutions $Z(T)$ of system (1.1), (1.2) is bounded in the space $W^{1, \infty}\left(T ; \mathbb{R}^{n_{1}+n_{2}}\right)=\left\{z(\cdot) \in L_{2}\left(T ; \mathbb{R}^{n_{1}+n_{2}}\right): \dot{z}(\cdot) \in L_{\infty}\left(T ; \mathbb{R}^{n_{1}+n_{2}}\right)\right\}$.

Lemma 3. The bundle of solutions of system $(2.1)$ is bounded in the space $W^{1, \infty}\left(T ; \mathbb{R}^{n_{2}}\right)$.

The validity of Lemmas 2 and 3 is easily verified by using conditions (1.3), (1.7)-(1.9). Define

$$
\begin{gathered}
\lambda_{j}\left(t, x(\cdot), y(\cdot), w^{(1)}(\cdot), v^{h}(\cdot)\right)=\varepsilon(t)+\alpha_{j} \int_{t_{j}}^{t}\left\{\left|v^{h}(\nu)\right|^{2}-|x(\nu)|^{2}\right\} d \nu, \\
\varepsilon(t)=\left|y(t)-w^{(1)}(t)\right|^{2}, \quad j \in\left[0: j_{*}\right], \quad t \in T .
\end{gathered}
$$

Lemma 4. Strategy (2.2) provides the inequality

$$
\lambda_{j}\left(t, x(\cdot), y(\cdot), w^{(1)}(\cdot), v^{h}(\cdot)\right) \leq b_{j}, \quad t \in \Delta^{(j)} \cap T, \quad j \in\left[0: j_{*}\right],
$$

where

$$
b_{j}=\left|y\left(t_{j}\right)-w^{(1)}\left(t_{j}\right)\right|^{2}+c_{j}^{(1)}(h+\delta)+c_{j}^{(2)} \sum_{k=j-l}^{j} \nu^{(k)}, \quad \nu^{(j)}=\left|v^{h}(\cdot)-x(\cdot)\right|_{L_{2}\left(\Delta^{(j-1)} ; \mathbb{R}^{\left.n_{1}\right)}\right.}^{2},
$$

$v^{h}(t)=x(t)$ for $t \in\left[t_{0}-\tau, t_{0}\right], v^{h}(t)=x_{0}(-\tau)$ for $t \in\left[t_{0}-\tau-\tau_{1}^{x}, t_{0}-\tau\right)$, and the constants $c_{j}^{(1)}$ and $c_{j}^{(2)}$ can be written explicitly.

Proof. Let us estimate the value

$$
\varepsilon_{j}(t)=\varepsilon(t)+\alpha_{j} \int_{t_{j}}^{t}\left\{\left|v^{h}(\nu)\right|^{2}-|x(\nu)|^{2}\right\} d \nu, \quad t \in \Delta^{(j)} \cap T .
$$

Fix $\tau_{i} \in \Delta^{(j)}$. Then, for $t \in \Delta^{(j)} \cap \delta_{i}=\left[\tau_{i}, \tau_{i+1}\right]$, we have

where

$$
\varepsilon_{j}(t) \leq \varepsilon_{j}\left(\tau_{i}\right)+\sum_{j=1}^{4} \Lambda_{j i}(t)
$$

$$
\begin{aligned}
& \Lambda_{1 i}(t)=2\left(s_{i}, \int_{\tau_{i}}^{t}\left\{\psi_{1}\left(\nu, x_{\nu}(s), y_{\nu}(s)\right)-\psi_{1}\left(\tau_{i}, v_{\nu}^{h}(s), \xi_{\tau_{i}}^{h}(s)\right)\right\} d \nu\right), \quad s_{i}=y\left(\tau_{i}\right)-w^{(1)}\left(\tau_{i}\right), \\
& \Lambda_{2 i}(t)=2\left(s_{i}, \int_{\tau_{i}}^{t}\left\{\psi_{2}\left(\nu, y_{\nu}(s)\right) x(\nu)-\psi_{2}\left(\tau_{i}, \xi_{\tau_{i}}^{h}(s)\right) v_{i}^{h}\right\} d \nu\right)+\alpha_{j} \int_{\tau_{i}}^{t}\left\{\left|v^{h}(\nu)\right|^{2}-|x(\nu)|^{2}\right\} d \tau,
\end{aligned}
$$

PROCEEDINGS OF THE STEKLOV INSTITUTE OF MATHEMATICS $\quad$ Vol. $280 \quad$ Suppl. $1 \quad 2013$ 


$$
\Lambda_{3 i}(t)=-2\left(t-\tau_{i}\right)\left(s_{i}, \xi^{h}\left(\tau_{i}\right)-w^{(1)}\left(\tau_{i}\right)\right), \quad \Lambda_{4 i}(t)=\left(t-\tau_{i}\right) \int_{\tau_{i}}^{t}\left|\dot{w}^{(1)}(\tau)-\dot{y}(\tau)\right|^{2} d \tau .
$$

By Lemmas 2 and 3,

$$
\Lambda_{4 i}(t) \leq K_{*}^{(j)}\left(t-\tau_{i}\right)^{2}, \quad t \in \delta_{i} .
$$

Note that $v^{h}\left(\tau_{i}+s\right)=v^{h}(t+s)$ for $s \geq t_{0}-\tau_{i}$ and $t \in\left[\tau_{i}, \tau_{i+1}\right]$; in addition,

$$
\left|\xi^{h}\left(\tau_{i}+s\right)-y(t+s)\right| \leq K_{*}\left(h+t-\tau_{i}\right) \quad \text { for } \quad \tau_{i}+s \geq t_{0}-\tau .
$$

Therefore, using Lemma 2, the Lipschitz property of the functions $x_{0}(s)$ and $y_{0}(s)$, and inequalities (1.9) and (2.12), we obtain the following relation for $t \in \delta_{i}$ :

$$
\begin{gathered}
\int_{\tau_{i}}^{t}\left|\psi_{1}\left(\nu, x_{\nu}(s), y_{\nu}(s)\right)-\psi_{1}\left(\tau_{i}, v_{\nu}^{h}(s), \xi_{\tau_{i}}^{h}(s)\right)\right| d \nu \\
\leq K_{*}^{(j)} \int_{\tau_{i}}^{t}\left\{\left(\nu-\tau_{j}\right)+\sum_{k=1}^{m}\left|x\left(\nu-\tau_{k}^{x}\right)-v^{h}\left(\nu-\tau_{k}^{x}\right)\right|+\sum_{k=0}^{n}\left|y\left(\nu-\tau_{k}^{y}\right)-\xi^{h}\left(\tau_{i}-\tau_{k}^{y}\right)\right|\right\} d \nu \\
\leq K_{0}^{(j)}\left\{\left(t-\tau_{i}\right)^{2}+\int_{\tau_{i}}^{t} \sum_{k=0}^{n}\left|y\left(\nu-\tau_{k}^{y}\right)-\xi^{h}\left(\tau_{i}-\tau_{k}^{y}\right)\right| d \nu+\int_{\tau_{i}}^{t}\left(\sum_{k=1}^{m}\left|x\left(\nu-\tau_{k}^{x}\right)-v^{h}\left(\nu-\tau_{k}^{x}\right)\right|\right) d \nu\right\} \\
\leq K_{1}^{(j)}\left(t-\tau_{i}\right)\left(h+t-\tau_{i}\right)+K_{2}^{(j)}\left(t-\tau_{i}\right)^{1 / 2} \sum_{k=1}^{m}\left(\int_{\tau_{i}-\tau_{k}^{x}}^{t-\tau_{k}^{x}}\left|x(\nu)-v^{h}(\nu)\right|^{2} d \nu\right)^{1 / 2},
\end{gathered}
$$

where $\tau_{0}^{y}=0$. Thus, for $t \in \delta_{i}$, we have the estimate

$$
\Lambda_{1 i}(t) \leq 2\left(t-\tau_{i}\right)\left|y\left(\tau_{i}\right)-w^{(1)}\left(\tau_{i}\right)\right|^{2}+K_{3}^{(j)}\left\{\left(t-\tau_{i}\right)\left(h+t-\tau_{i}\right)^{2}+\sum_{k=1}^{m} \int_{\tau_{i}-\tau_{k}^{x}}^{t-\tau_{k}^{x}}\left|x(\nu)-v^{h}(\nu)\right|^{2} d \nu\right\} .
$$

Further, in view of (1.4), we conclude that

$$
\Lambda_{3 i}(t) \leq-2\left(t-\tau_{i}\right)\left|y\left(\tau_{i}\right)-w^{(1)}\left(\tau_{i}\right)\right|^{2}+K_{4}^{(j)} h\left(t-\tau_{i}\right), \quad t \in \delta_{i} .
$$

Note that (1.4), (1.8), and (2.12) imply the inequalities

$$
\left|\psi_{2}\left(\nu, y_{\nu}(s)\right) x(\nu)-\psi_{2}\left(\tau_{i}, \xi_{\tau_{i}}^{h}(s)\right) x(\nu)\right| \leq\left|\psi_{2}\left(\nu, y_{\nu}(s)\right)-\psi_{2}\left(\tau_{i}, \xi_{\tau_{i}}^{h}(s)\right)\right||x(\nu)| \leq K_{0}\left(h+\nu-\tau_{i}\right)
$$

for $\nu \in\left[\tau_{i}, \tau_{i+1}\right]$. Thus,

$$
\Lambda_{2 i}(t) \leq K_{5}^{(j)}\left(t-\tau_{i}\right)\left(h+t-\tau_{i}\right)+\int_{\tau_{i}}^{t}\left\{2\left(l_{i}, \psi_{2}\left(\tau_{i}, \xi_{\tau_{i}}^{h}(s)\right)\left\{v_{i}^{h}-x(\nu)\right\}+\alpha_{j}\left\{\left|v_{i}^{h}\right|^{2}-|x(\nu)|^{2}\right\}\right\} d \nu .\right.
$$

By the choice of the control $v_{i}^{h}$ and strategy $V\left(\tau_{i}, w_{\tau_{i}}(s), \xi_{\tau_{i}}^{h}(s)\right)$ (see (1.12) and (2.2)), we get

$$
\Lambda_{2 i}(t) \leq K_{5}^{(j)}\left(t-\tau_{i}\right)\left(h+t-\tau_{i}\right)
$$


Combining (2.10)-(2.15), we obtain for $t \in \Delta^{(j)} \cap \delta_{i}$

$$
\varepsilon_{j}(t) \leq \varepsilon_{j}\left(\tau_{i}\right)+K_{6}^{(j)} \delta(h+\delta)+K_{3}^{(j)} \sum_{k=1}^{m} \int_{\tau_{i}-\tau_{k}^{x}}^{t-\tau_{k}^{x}}\left|x(\nu)-v^{h}(\nu)\right|^{2} d \nu
$$

Thus, for $t \in \Delta^{(j)}=\left[t_{j}, t_{j+1}\right]$,

$$
\varepsilon_{j}(t) \leq \varepsilon_{j}\left(t_{j}\right)+K_{7}^{(j)}(h+\delta)+K_{8}^{(j)} \sum_{k=1}^{m} \int_{t_{j}-\tau_{k}^{x}}^{t-\tau_{k}^{x}}\left|x(\nu)-v^{h}(\nu)\right|^{2} d \nu
$$

Hence,

$$
\varepsilon_{j}(t) \leq \varepsilon_{j}\left(t_{j}\right)+K_{7}^{(j)}(h+\delta)+K_{9}^{(j)} \int_{t_{j}-\tau}^{t_{j+1}-\tau_{1}^{x}}\left|x(\nu)-v^{h}(\nu)\right|^{2} d \nu
$$

Note that $\tau=l \tau_{1}^{x}+\gamma$ and $\gamma \geq 0$. Therefore, $t_{j+1}-\tau_{1}^{x}=t_{j}$ and $t_{j-l-1} \leq t_{j}-\tau \leq t_{j-l}$. Thus, for $t \in \Delta^{(j)}$, we have

$$
\varepsilon_{j}(t) \leq \varepsilon_{j}\left(t_{j}\right)+K_{7}^{(j)}(h+\delta)+K_{9}^{(j)} \int_{t_{j-l-1}}^{t_{j}}\left|x(\nu)-v^{h}(\nu)\right|^{2} d \nu=\varepsilon_{j}\left(t_{j}\right)+K_{7}^{(j)}(h+\delta)+K_{9}^{(j)} \sum_{k=j-l}^{j} \nu^{(k)} .
$$

Here, the constants $K_{k}^{(j)}, k \in[0: 9]$, can be specified explicitly. Thus, we can assume $c_{j}^{(1)}=K_{7}^{(j)}$, $c_{j}^{(2)}=K_{9}^{(j)}$. The lemma is proved.

Lemma 5. Suppose that $\delta \leq h$ and the values $\alpha_{j}$ are specified according to (2.3). Then,

$$
\begin{gathered}
\nu^{(j)} \leq c_{j} g_{j}(h), \\
b_{j} \leq c_{j}^{(0)} g_{j}(h) .
\end{gathered}
$$

Proof. For simplicity, let $t_{j_{*}+1}=\vartheta$. By Lemma 4 , for $t \in \Delta^{(j)}$, we have

$$
\begin{gathered}
\left|y(t)-w^{(1)}(t)\right|=\varepsilon^{1 / 2}(t) \leq\left(\lambda_{j}\left(t, x(\cdot), y(\cdot), w^{(1)}(\cdot), v^{h}(\cdot)\right)\right. \\
\left.+\alpha_{j} \int_{t_{j}}^{t}\left\{\left|v^{h}(\nu)\right|^{2}+|x(\nu)|^{2}\right\} d \nu\right)^{1 / 2} \leq\left(b_{j}+\alpha_{j} \rho_{A}\right)^{1 / 2},
\end{gathered}
$$

where $\rho_{A}=2 \tau_{*} d^{2}(A), d(A)=\sup \{|u|: u \in S(A)\}$. Since $t_{j} \in \Delta_{h}$, it follows that, for each $j \in\left[0: j_{*}\right]$, there exists $i=i_{j}(h)$ such that $t_{j}=\tau_{i_{j}(h)}$. Define $\varrho_{j} \equiv\left|f_{1}(\cdot)-F_{1}(\cdot)\right|_{L_{2}\left(\Delta^{(j)} ; \mathbb{R}^{\left.n_{2}\right)}\right.}^{2}$. Then, in view of Lemma $2,(1.9)$, and (2.12),

$$
\varrho_{j} \leq d_{j}^{(1)} \sum_{i=i_{j}(h)}^{i=i_{j+1}(h)-1} \int_{\tau_{i}}^{\tau_{i+1}}\left\{\delta^{2}+h^{2}+\gamma^{h}(\nu)+\gamma_{i}^{h}(\nu)+\left|\xi^{h}\left(\tau_{i}\right)-w^{(1)}\left(\tau_{i}\right)\right|^{2}\right\} d \nu
$$


where

$$
\gamma^{h}(\nu)=\sum_{k=1}^{m}\left|x\left(\nu-\tau_{k}^{x}\right)-v^{h}\left(\nu-\tau_{k}^{x}\right)\right|^{2}, \quad \gamma_{i}^{h}(\nu)=\sum_{k=0}^{n}\left|y\left(\nu-\tau_{k}^{y}\right)-\xi^{h}\left(\tau_{i}-\tau_{k}^{y}\right)\right|^{2} .
$$

Note that the following inequalities hold:

$$
\begin{gathered}
\int_{t_{j}}^{t_{j+1}} \gamma^{h}(\nu) d \nu \leq d_{j}^{(2)} \int_{t_{j}-\tau}^{t_{j}}\left|x(\nu)-v^{h}(\nu)\right|^{2} d \nu \leq d_{j}^{(2)} \int_{t_{j-l-1}}^{t_{j}}\left|x(\nu)-v^{h}(\nu)\right|^{2} d \nu=d_{j}^{(2)} \sum_{k=j-l}^{j} \nu^{(k)}, \\
\int_{t_{j}}^{t_{j+1}} \gamma_{i}^{h}(\nu) d \nu \leq d_{j}^{(3)}\left(h^{2}+\delta^{2}\right) .
\end{gathered}
$$

In addition,

$$
\nu^{(k)}=0 \quad \text { for } \quad k \in[-l: 0] .
$$

Then, in view of (2.18)-(2.20), we obtain the estimates

$$
\varrho_{j} \leq d_{j}^{(5)}\left\{h^{2}+\delta^{2}+\sum_{k=j-l}^{j} \nu^{(k)}+b_{j}+\alpha_{j}\right\}, \quad j \in\left[0: j_{*}\right]
$$

It is easy to see that

$$
\left|f_{2}(\cdot)-F_{2}(\cdot)\right|_{L_{2}\left(\Delta^{(j)} ; \mathbb{R}^{\left.n_{2} \times n_{1}\right)}\right.}^{2} \leq d_{j}^{(5)}\left(h^{2}+\delta^{2}\right), \quad j \in\left[0: j_{*}\right]
$$

Here, the constants $d_{j}^{(1)}-d_{j}^{(5)}$ can be specified explicitly. By Lemma $4,(2.18)$, and (2.21), for $\delta \leq h$, we obtain

$$
\begin{gathered}
\lambda_{0}\left(t, x(\cdot), y(\cdot), w^{(1)}(\cdot), v^{h}(\cdot)\right) \leq b_{0} \leq c_{0}^{*} h, \quad t \in \Delta^{(0)}, \\
\left|y\left(t_{1}\right)-w^{(1)}\left(t_{1}\right)\right|^{2} \leq \rho_{A} \alpha_{0}+c_{0}^{*} h \leq c_{*} h^{2 / 3} .
\end{gathered}
$$

Next, in view of (2.21)-(2.24), for $h \in(0,1)$, we have

$$
\varrho_{0} \leq d_{0}^{(1)}\left\{h^{2}+\delta^{2}+b_{0}+h^{2 / 3}\right\} \leq d_{0}^{*} h^{2 / 3}, \quad\left|f_{2}(\cdot)-F_{2}(\cdot)\right|_{L_{2}\left(\Delta^{(0)} ; \mathbb{R}^{\left.n_{2} \times n_{1}\right)}\right.}^{2} \leq c_{j}^{(*)} h^{2} .
$$

By Condition 1, we can use Lemma 1. Write $p=y, q=w^{(1)}, u_{1}=x, u_{2}=v^{h}, f_{1}(t)=$ $\psi_{1}\left(t, x_{t}(s), y_{t}(s)\right), f_{2}(t)=\psi_{2}\left(t, y_{t}(s)\right), F_{1}(t)=\psi_{1}\left(\tau_{i}, v_{\tau_{i}}^{h}(s), \xi_{\tau_{i}}^{h}(s)\right)+2\left(\xi^{h}\left(\tau_{i}\right)-w^{(1)}\left(\tau_{i}\right)\right)$, and $F_{2}(t)=$ $\psi_{2}\left(\tau_{i}, \xi_{\tau_{i}}^{h}(s)\right)$ for $t \in\left[\tau_{i}, \tau_{i+1}\right)$. Then, taking $a_{1}^{(0)}=d_{0}^{*} h^{2 / 3}, a_{2}^{(0)}=c_{j}^{(*)} h^{2}, a_{3}^{(0)}=c_{0}^{*} h, a_{4}^{(0)}=c_{*} h^{2 / 3}$, and $\tilde{\alpha}_{0}=\alpha_{0}=c h^{2 / 3}$, we get

$$
\nu^{(1)}=\left|x(\cdot)-v^{h}(\cdot)\right|_{L_{2}\left(\Delta^{(0)} ; \mathbb{R}^{\left.n_{1}\right)}\right.}^{2} \leq \tilde{c}_{1} h^{1 / 3}=c_{1} g_{1}(h) ;
$$

i.e., inequality (2.16) is valid for $j=1$. Further, in view of (2.25) and (2.26), we derive

$$
b_{1}=\left|y\left(t_{1}\right)-w^{(1)}\left(t_{1}\right)\right|^{2}+c_{1}^{(1)}(h+\delta)+c_{1}^{(2)} \sum_{k=1-l}^{1} \nu^{(k)} \leq \tilde{c}_{1}^{(0)} h^{1 / 3}=c_{1}^{(0)} g_{1}(h) .
$$


Inequality (2.17) for $j=1$ is also established. From (2.18), we get the inequalities

$$
\left|y\left(t_{j}\right)-w^{(1)}\left(t_{j}\right)\right|^{2} \leq b_{j-1}+\rho_{A} \alpha_{j-1}, \quad j \in\left[1: j_{*}-1\right] .
$$

Consequently, using (2.28) and the rule for choosing $b_{j}$, we obtain

$$
b_{j} \leq b_{j-1}+\rho_{A} \alpha_{j-1}+c_{j}^{(1)}(h+\delta)+c_{j}^{(2)} \sum_{k=j-l}^{j} \nu^{(k)} \leq b_{j-1}+d_{j}\left(h+\alpha_{j-1}+\sum_{k=j-l}^{j} \nu^{(k)}\right),
$$

where $d_{j}=$ const $\in(0,+\infty)$. For $j \geq 1$, write in Lemma $1 a_{1}^{(j)}=d_{j}^{(4)}\left\{h^{2}+\delta^{2}+\sum_{k=j-l}^{j} \nu^{(k)}+\right.$ $\left.a_{3}^{(j)}+\alpha_{j}\right\}, a_{3}^{(j)}=b_{j}, a_{2}^{(j)}=d_{j}^{(5)}\left(h^{2}+\delta^{2}\right)$, and $a_{4}^{(j)}=b_{j-1}+\rho_{A} \alpha_{j-1}$ for $j \in\left[1: j_{*}\right]$. (In the choice of the values $a_{i}^{(j)}$, we have used Lemma 4 and inequalities (2.23), (2.24), and (2.28).) Then, from this lemma and (2.29), we obtain

$$
\nu^{(j+1)} \leq c^{(j)}\left\{h^{1 / 2}+\left(\sum_{k=j-l}^{j} \nu^{(k)}\right)^{1 / 2}+b_{j-1}^{1 / 2}+\alpha_{j-1}^{1 / 2}+\alpha_{j}^{1 / 2}\right\}+b_{j} \alpha_{j}^{-1}, \quad j \in\left[1: j_{*}\right] .
$$

Now, we can prove (2.16) and (2.17) by induction. For $j=1$, inequalities (2.16) and (2.17) are true (see (2.26), (2.27)). Setting $j=1$ in (2.30), we find that $(2.16)$ is valid for $j=2$. This and (2.29) yield inequality (2.17) for $j=2$. Assume that inequalities (2.16) and (2.17) hold for $j>2$. By the relations $h \in(0,1), g_{j}(h)<g_{j+1}(h)$, and $g_{j+1}(h)=g_{j}^{1 / 3}(h)$, we have

$$
\left(\sum_{k=j-l}^{j} \nu^{(k)}\right)^{1 / 2} \leq l\left(\nu^{(j)}\right)^{1 / 2} \leq l g_{j}^{1 / 3}(h)=l g_{j+1}(h)
$$

Hence, in view of $(2.30)$ and the inequalities $b_{j-1} \leq c_{j-1}^{(0)} g_{j-1}(h) \leq c_{j-1}^{(0)} g_{j+1}(h)$ and $\alpha_{j}<\alpha_{j+1}$, we have

$$
\nu^{(j+1)} \leq c_{j+1}\left\{g_{j+1}(h)+\alpha_{j}^{1 / 2}(h)\right\}+g_{j}(h) \alpha_{j}^{-1}(h) .
$$

Using the equality $g_{j}(h) \alpha_{j}^{-1}(h)=1 / C g_{j}^{1 / 3}(h)$ (see $(2.3)$ ), we derive (2.16) from (2.31). Inequality (2.17) is derived similarly from (2.29) and (2.16). The lemma is proved.

\section{RECONSTRUCTION OF UNKNOWN CONTROLS}

Let us describe the algorithm for the dynamic reconstruction of the unknown input $u(\cdot)$. We will specify the rules for choosing the strategy $U$ (1.13) and the model. This will allow us to construct the function $u^{h}(\cdot)$, which approximates $u(\cdot)$ (see (1.6)).

Fix the value of measurement error $h \in(0,1)$ and the family of partitions $\Delta_{h}$ (1.10) of the interval $T$. Consider a model described by the equation

$$
\begin{gathered}
\dot{w}^{(0)}(t)=F_{0}\left(\tau_{i}, v_{\tau_{i}}^{h}(s), \xi_{\tau_{i}}^{h}(s), u_{i}^{h}\right), \\
\dot{w}^{(1)}(t)=F_{1}\left(\tau_{i}, v_{\tau_{i}}^{h}(s), \xi_{\tau_{i}}^{h}(s), w^{(1)}\left(\tau_{i}\right)\right),
\end{gathered}
$$

where $F_{0}\left(\tau_{i}, v_{\tau_{i}}^{h}(s), \xi_{\tau_{i}}^{h}(s), u_{i}^{h}\right)=f_{1}\left(\tau_{i}, v_{\tau_{i}}^{h}(s), \xi_{\tau_{i}}^{h}(s)\right)+f_{2}\left(\tau_{i}, v_{\tau_{i}}^{h}(s), \xi_{\tau_{i}}^{h}(s)\right) u_{i}^{h}, w^{(0)} \in \mathbb{R}^{n_{1}}$, and $t \in$ $\left[\tau_{i}, \tau_{i+1}\right)$, with initial condition $w_{t_{0}}^{(0)}(s)=x\left(t_{0}+s\right)$ for $s \in[-\tau, 0], w_{t_{0}}^{(1)}(s)=y\left(t_{0}+s\right)$ for $s \in[-\tau, 0)$, 
and $w^{(1)}\left(t_{0}\right)=\xi^{h}\left(t_{0}\right)$. The solution $w^{h}(\cdot)=\left\{w^{(0)}(\cdot), w^{(1)}(\cdot)\right\}=w^{h}\left(\cdot ; t_{0}, w_{t_{0}}^{h}(s), v^{h}(\cdot), u^{h}(\cdot)\right)$ of this equation is also understood in the sense of Carathéodory.

Thus, in the equation of the model (see (1.11)),

$$
\rho\left(\tau_{i}, v_{\tau_{i}}^{h}(s), \xi_{\tau_{i}}^{h}(s), u_{i}^{h}, w^{(1)}\left(\tau_{i}\right)\right)=\left\{\begin{array}{c}
F_{0}\left(\tau_{i}, v_{\tau_{i}}^{h}(s), \xi_{\tau_{i}}^{h}(s), u_{i}^{h}\right) \\
F_{1}\left(\tau_{i}, v_{\tau_{i}}^{h}(s), \xi_{\tau_{i}}^{h}(s), w^{(1)}\left(\tau_{i}\right)\right)
\end{array}\right\} .
$$

The strategy $U(1.13)$ is specified as follows:

$$
U\left(\tau_{i}, w_{\tau_{i}}^{(0)}(s), \xi_{\tau_{i}}^{h}(s), v_{\tau_{i}}^{h}(s)\right)=\arg \min \left\{2\left(l_{i}^{(1)}, f_{2}\left(\tau_{i}, v_{\tau_{i}}^{h}(s), \xi_{\tau_{i}}^{h}(s)\right) u\right)+\alpha^{(1)}|u|^{2}: u \in P\right\} .
$$

Here, $\alpha^{(1)}=\alpha^{(1)}(h):(0,1) \rightarrow R_{+}$is some function and $l_{i}^{(1)}=w^{(0)}\left(\tau_{i}\right)-v_{i}^{h}$.

Let $U(y(\cdot))$ denote the set of all controls $u(\cdot) \in P(\cdot)$ corresponding to the output $y(\cdot)$. It is easy to verify that this set is convex, bounded, and closed in $L_{2}\left(T ; \mathbb{R}^{r}\right)$. Therefore, there exists an element

$$
u_{*}(\cdot)=u_{*}(\cdot ; y(\cdot))=\arg \min \left\{|u(\cdot)|_{L_{2}\left(T ; \mathbb{R}^{r}\right)}: u(\cdot) \in U(y(\cdot))\right\} .
$$

Theorem 2. Suppose that $\delta=\delta(h) \leq h, \alpha^{(1)}(h) \rightarrow 0$, and $g_{j_{*}}^{1 / 2}(h) / \alpha^{(1)}(h) \rightarrow 0$ as $h \rightarrow 0$. Then,

$$
u^{h}(\cdot) \rightarrow u_{*}(\cdot) \quad \text { in } \quad L_{2}\left(T ; \mathbb{R}^{r}\right) \quad \text { as } \quad h \rightarrow 0
$$

Let us first prove two auxiliary statements.

Lemma 6. The bundle of solutions of system (3.1) is bounded in the space $W^{1, \infty}\left(T ; \mathbb{R}^{n_{1}+n_{2}}\right)$.

The validity of Lemma 6 is easily verified by using conditions (1.3), (1.4), and (1.7)-(1.9).

Introduce the value

$$
\lambda(t)=\lambda\left(t, x(\cdot), w^{(0)}(\cdot), u_{*}(\cdot), u^{h}(\cdot)\right)=\left|x(t)-w^{(0)}(t)\right|^{2}+\alpha^{(1)} \int_{t_{0}}^{t}\left\{\left|u^{h}(\nu)\right|^{2}-\left|u_{*}(\nu)\right|^{2}\right\} d \nu .
$$

Lemma 7. Strategy (3.2) provides the inequality

$$
\lambda\left(t, x(\cdot), w^{(0)}(\cdot), u_{*}(\cdot), u^{h}(\cdot)\right)<\left|x\left(t_{0}\right)-w^{(0)}\left(t_{0}\right)\right|^{2}+C^{(1)}(h+\delta)+C^{(2)} \int_{t_{0}}^{t}\left|x(\nu)-v^{h}(\nu)\right| d \nu
$$

where the constants $C^{(1)}$ and $C^{(2)}$ can be specified explicitly.

Proof. Let us estimate $\lambda(t)$. Fix $\tau_{i}$. Then, for $t \in \delta_{i}=\left[\tau_{i}, \tau_{i+1}\right]$,

$$
\lambda(t) \leq \lambda\left(\tau_{i}\right)+\sum_{j=1}^{3} \Lambda_{j i}^{0}(t)
$$

where

$$
\Lambda_{1 i}^{0}(t)=2\left(s_{i}^{(1)}, \int_{\tau_{i}}^{t}\left\{f_{1}\left(\nu, x_{\nu}(s), y_{\nu}(s)\right)-f_{1}\left(\tau_{i}, v_{\tau_{i}}^{h}(s), \xi_{\tau_{i}}^{h}(s)\right)\right\} d \nu\right), \quad s_{i}^{(1)}=x\left(\tau_{i}\right)-w^{(0)}\left(\tau_{i}\right)
$$




$$
\begin{gathered}
\Lambda_{2 i}^{0}(t)=2\left(s_{i}^{(1)}, \int_{\tau_{i}}^{t}\left\{f_{2}\left(\nu, x_{\nu}(s), y_{\nu}(s)\right) u_{*}(\nu)-f_{2}\left(\tau_{i}, v_{\tau_{i}}^{h}(s), \xi_{\tau_{i}}^{h}(s)\right) u_{i}^{h}\right\} d \nu\right) \\
+\alpha^{(1)} \int_{\tau_{i}}^{t}\left\{\left|u^{h}(\nu)\right|^{2}-\left|u_{*}(\nu)\right|^{2}\right\} d \nu, \quad \Lambda_{3 i}^{0}(t)=\left(t-\tau_{i}\right) \int_{\tau_{i}}^{t}\left|\dot{w}^{(0)}(\tau)-\dot{x}(\tau)\right|^{2} d \tau .
\end{gathered}
$$

By Lemmas 2 and 6,

$$
\begin{gathered}
\Lambda_{3 i}^{0}(t) \leq k_{0}\left(t-\tau_{i}\right)^{2}, \quad t \in \delta_{i}, \\
\left|s_{i}^{(1)}\right| \leq k_{1} .
\end{gathered}
$$

Here and below, the constants $k_{j}, j=0,1, \ldots$, are independent of $i$ and $t$. Since $x_{0}(s)$ and $y_{0}(s)$ are Lipschitz functions, we have, for $t \in \delta_{i}$, the relations

$$
\begin{gathered}
\int_{\tau_{i}}^{t}\left|f_{1}\left(\nu, x_{\nu}(s), y_{\nu}(s)\right)-f_{1}\left(\tau_{i}, v_{\nu}^{h}(s), \xi_{\tau_{i}}^{h}(s)\right)\right| d \nu \\
\leq k_{2} \int_{\tau_{i}}^{t}\left\{\left(\nu-\tau_{j}\right)+\sum_{k=1}^{m}\left|x\left(\nu-\tau_{k}^{x}\right)-v^{h}\left(\nu-\tau_{k}^{x}\right)\right|+\sum_{k=0}^{n}\left|y\left(\nu-\tau_{k}^{y}\right)-\xi^{h}\left(\tau_{i}-\tau_{k}^{y}\right)\right|\right\} d \nu \\
\leq k_{3}\left(t-\tau_{i}\right)\left(h+\left(t-\tau_{i}\right)\right)+k_{4} \int_{\tau_{i}}^{t}\left(\sum_{k=0}^{m}\left|x\left(\nu-\tau_{k}^{x}\right)-v^{h}\left(\nu-\tau_{k}^{x}\right)\right|\right) d \nu, \quad \tau_{0}^{x}=0 .
\end{gathered}
$$

Thus, by (3.5), for $t \in \delta_{i}$, we have the estimate

$$
\Lambda_{1 i}^{0}(t) \leq k_{5}\left\{\left(t-\tau_{i}\right)\left(h+\left(t-\tau_{i}\right)\right)+\int_{\tau_{i}}^{t}\left(\sum_{k=0}^{m}\left|x\left(\nu-\tau_{k}^{x}\right)-v^{h}\left(\nu-\tau_{k}^{x}\right)\right|\right) d \nu\right\} .
$$

The following estimate is established for $t \in \delta_{i}$ similarly to (3.6):

$$
\begin{gathered}
\int_{\tau_{i}}^{t}\left|f_{2}\left(\eta, x_{\eta}(s), y_{\eta}(s)\right) u_{*}(\eta)-f_{2}\left(\tau_{i}, v_{\tau_{i}}^{h}(s), \xi_{\tau_{i}}^{h}(s)\right) u_{*}\left(\eta^{h}\right)\right| d \eta \\
\leq k_{6}\left\{\left(t-\tau_{i}\right)^{2}+\int_{\tau_{i}-\tau}^{t}\left|y(\tau)-\xi^{h}(\tau)\right| d \tau+\int_{\tau_{i}}^{t}\left(\sum_{k=0}^{m}\left|x\left(\nu-\tau_{k}^{x}\right)-v^{h}\left(\nu-\tau_{k}^{x}\right)\right|\right) d \nu\right\} .
\end{gathered}
$$

In addition, in view of Lemma 2 and inequality (1.4), for $t \in \delta_{i}$, we obtain

$$
\int_{\tau_{i}-\tau}^{t}\left|y(\tau)-\xi^{h}(\tau)\right| d \tau \leq k_{7}(h+\delta)
$$


Therefore, by (3.7) and (3.8), we have for $t \in \delta_{i}$

$$
\begin{gathered}
\Lambda_{2 i}^{0}(t) \leq k_{8}\left(t-\tau_{i}\right)(h+\delta)+\int_{\tau_{i}}^{t}\left\{2 \left(s_{i}^{(1)}, f_{2}\left(\tau_{i}, v_{\tau_{i}}^{h}(s), \xi_{\tau_{i}}^{h}(s)\right)\left\{u_{*}(\nu)-u_{i}^{h}\right\}\right.\right. \\
\left.+\alpha^{(1)}\left\{\left|u_{i}^{h}\right|^{2}-\left|u_{*}(\nu)\right|^{2}\right\}\right\} d \nu+k_{9}\left|s_{i}^{(1)}\right| \int_{\tau_{i}}^{t}\left(\sum_{k=0}^{m}\left|x\left(\nu-\tau_{k}^{x}\right)-v^{h}\left(\nu-\tau_{k}^{x}\right)\right|\right) d \nu .
\end{gathered}
$$

Thus, using Lemma 2, we establish for $t \in \delta_{i}$ the estimate

$$
\int_{\tau_{i}}^{t}\left|l_{i}^{(1)}-s_{i}^{(1)}\right| d \nu=\int_{\tau_{i}}^{t}\left|x\left(\tau_{i}\right)-v_{i}^{h}\right| d \nu \leq \int_{\tau_{i}}^{t}\left|x(\nu)-v^{h}(\nu)\right| d \nu+k_{10}\left(t-\tau_{i}\right) .
$$

Since the control $u_{i}^{h}$ and the strategy $U\left(\tau_{i}, w_{\tau_{i}}^{h}(s), \xi_{\tau_{i}}^{h}(s), v_{\tau_{i}}^{h}(s)\right)$ were chosen according to rules (1.13) and (3.2), we obtain from (3.9) and (3.10) the following estimate for $t \in \delta_{i}$ :

$$
\Lambda_{2 i}^{0}(t) \leq k_{11}\left(t-\tau_{i}\right)(h+\delta)+k_{12} \int_{\tau_{i}}^{t}\left(\sum_{j=0}^{m}\left|x\left(\nu-\tau_{j}^{x}\right)-v^{h}\left(\nu-\tau_{j}^{x}\right)\right|\right) d \nu .
$$

Combining estimates (3.3), (3.4), (3.6), and (3.11), we obtain for $t \in \delta_{i}$

$$
\lambda(t) \leq \lambda\left(\tau_{i}\right)+k_{13} \delta(h+\delta)+k_{14} \int_{\tau_{i}}^{t}\left(\sum_{j=0}^{m}\left|x\left(\nu-\tau_{j}^{x}\right)-v^{h}\left(\nu-\tau_{j}^{x}\right)\right|\right) d \nu .
$$

Thus, for $t \in T$,

$$
\begin{gathered}
\lambda(t) \leq \lambda\left(t_{0}\right)+k_{15}(h+\delta)+k_{15} \int_{t_{0}}^{t}\left(\sum_{j=1}^{m}\left|x\left(\nu-\tau_{j}^{x}\right)-v^{h}\left(\nu-\tau_{j}^{x}\right)\right|\right) d \nu \\
\leq k_{15}(h+\delta)+k_{16} \int_{t_{0}}^{t}\left|x(\nu)-v^{h}(\nu)\right|^{2} d \nu,
\end{gathered}
$$

because $v^{h}\left(t_{0}+s\right)=x\left(t_{0}+s\right)$ for $s \in[-\tau, 0]$. The statement of the lemma follows from (3.12). The lemma is proved.

Proof of Theorem 2. In view of Lemma 7, Theorem 1 , and the equality $w^{(0)}\left(t_{0}\right)=x\left(t_{0}\right)$, we have

$$
\lambda\left(t, x(\cdot), w^{(0)}(\cdot), u_{*}(\cdot), u^{h}(\cdot)\right) \leq c^{(3)}\left(h+\delta(h)+g_{j_{*}}^{1 / 2}(h)\right)
$$

Thus,

$$
\begin{gathered}
\sup _{t \in T}\left|x(t)-w^{(0)}(t)\right|^{2} \leq c^{(4)}\left(\alpha^{(1)}(h)+h+\delta(h)+g_{j_{*}}^{1 / 2}(h)\right), \\
\int_{t_{0}}^{t}\left|u^{h}(\nu)\right|^{2} d \nu \leq \int_{t_{0}}^{t}\left|u_{*}(\nu)\right|^{2} d \nu+\left(h+\delta(h)+g_{j_{*}}^{1 / 2}(h)\right) / \alpha^{(1)}(h), \quad t \in T .
\end{gathered}
$$

Further proof follows the standard scheme (see, for example, [6]). The theorem is proved.

PROCEEDINGS OF THE STEKLOV INSTITUTE OF MATHEMATICS $\quad$ Vol. $280 \quad$ Suppl. $1 \quad 2013$ 


\section{ACKNOWLEDGMENTS}

This work was supported by the Russian Foundation for Basic Research (project nos. 11-0112112-ofi-m-2011 and 12-01-00175-a), by the Presidium of the Ural Branch of the Russian Academy of Sciences (project nos. 12-P-1-1019 and 12-S-1-1017), and by the Program for State Support of Leading Scientific Schools of the Russian Federation (project no. NSh-6512.2012.1).

\section{REFERENCES}

1. A. N. Tikhonov and V. Ya. Arsenin, Solutions of Ill-Posed Problems (Nauka, Moscow, 1979; Wiley, New York, 1981) [in Russian].

2. M. M. Lavrent'ev, V. G. Romanov, and S. P. Shishatskii, Ill-Posed Problems of Mathematical Physics (Nauka, Novosibirsk, 1980; Amer. Math. Soc., Providence, RI, 1986).

3. F. P. Vasil'ev, Methods for Solving Extremal Problems (Nauka, Moscow, 1981) [in Russian].

4. V. K. Ivanov, V. V. Vasin, and V. P. Tanana, Theory of Linear Ill-Posed Problems and Its Applications (Nauka, Moscow, 1978; VSP, Utrecht, 2002).

5. H. T. Banks and K. Kunisch, Estimation Techniques for Distributed Parameter Systems (Birkhäuser, Boston, 1989).

6. A. V. Kryazhimskii and Yu. S. Osipov, Engrg. Cybernetics 21 (2), 38 (1984).

7. N. N. Krasovskii, The Theory of Motion Control (Nauka, Moscow, 1968) [in Russian].

8. N. N. Krasovskii and A. I. Subbotin, Positional Differential Games (Nauka, Moscow, 1974) [in Russian].

9. Yu. S. Osipov, in Functional-Differential Equations: Proc. Internat. Symposium, Kyoto, Japan, 1990 (World Scientific, Singapore, 1991), pp. 309-317.

10. M. S. Blizorukova, in Applied Mathematics and Information Science: Proceedings of the Faculty of Computational Mathematics and Cybernetics, Moscow State University (MAKS, Moscow, 2007), Issue 5, pp. $105-115$ [in Russian].

11. E. V. Vasil'eva, in Problems of Mathematical Physics (Dialog-MGU, Moscow, 1998), pp. 68-74 [in Russian].

12. A. M. Kadiev and V. I. Maksimov, Funct. Differ. Equ. 15 (3-4), 219 (2008).

13. F. Kappel and V. I. Maksimov, Int. J. Appl. Math. Comput. Sci. 10 (2), 283 (2000).

14. V. I. Maksimov, in Proc. 5th European Control Conf., Karlsruhe, Germany, 1999, pp. 721-727.

15. Yu. S. Osipov and A. V. Kryazhimskii, Inverse Problems for Ordinary Differential Equations: Dynamical Solutions (Gordon and Breach, London, 1995).

16. A. V. Kryazhimskii and Yu. S. Osipov, in Collection of Scientific Papers (Ural'sk. Otdel. Akad. Nauk SSSR, Sverdlovsk, 1989), pp. 33-47 [in Russian].

17. A. Yu. Vdovin, in Collection of Scientific Papers (Ural'sk. Otdel. Akad. Nauk SSSR, Sverdlovsk, 1986), pp. 3-11 [in Russian].

Translated by Ya. Salii 